\title{
New Version of the Bulgarian Emergency Response System in case of Nuclear Accident over Europe (BERS2)
}

\author{
D.Syrakov, M.Prodanova, K.Slavov, E.Georgieva \\ National Institute of Meteorology and Hydrology, 66, Tsarigradsko shosse Blvd., Sofia, Bulgaria \\ Corresponding author's e-mail address: dimiter.syrakov@meteo.bg
}

\begin{abstract}
A PC-oriented Emergency Response System in case of nuclear accident (BERS) has been developed and works operationally at the Bulgarian National Institute of Meteorology and Hydrology (NIMH). The creation and the development of BERS was highly stimulated by the ETEX (European Tracer EXperiment) project. BERS comprises two main parts - operational and accidental ones. The operational part runs automatically every day. It prepares the input meteorological file used by both trajectory and dispersion models, runs the trajectory model for 35 European Nuclear Power Plants (NPP), visualizes the results and uploads the maps of trajectories to a dedicated web-site. The accidental part is activated manually when a real radioactive release occurs or during emergency exercises. Its core is the Bulgarian dispersion model EMAP. Outputs are time sequences of concentration and accumulated deposition fields. In the paper, the new version of BERS regarding mainly the operational part is presented. The main improvement is in the simulation of concentration and deposition fields applying mesometeorological and dispersion models in the operational part, as supplement to trajectory calculations.
\end{abstract}

Key words: nuclear accident, emergency response, early warning system, air dispersion models

\section{Introduction}

The industrial accidents causing a release of harmful (chemical or radioactive) material to the atmosphere can have consequences extending to hundreds and even thousands of kilometers. In such a case the decision-makers need information about the possible long-range transport of pollution over the country of interest. For that purpose computer-based Emergency Response Systems have been established in many countries, simulating and predicting the distribution of the released pollution.

Such system has been developed and works operationally at the National Institute of Meteorology and Hydrology (NIMH) of Bulgaria. Its creation and development was highly stimulated by the European Tracer Experiment (ETEX) (Graziani et al., 1998). NIMH took part in all activities of ETEX. The main results connected with ETEX are given in Syrakov and Prodanova (1998). The dispersion model EMAP, developed ad hoc for this purpose, was tested and has shown good performance. Full description of the first version of BERS is given in Syrakov et al. (2003). During the years BERS took part in many international projects on model evaluation and model inter-comparison (ETEX, RTMOD, ENSEMBLE).
After some modifications, BERS was used in many other study cases like the pollution during the wars in Yugoslavia and Iraq, the analysis of some local industrial accidents, the consequences of the Icelandic volcano eruption in 2010 and the Fukushima nuclear accident in 2011. A significant upgrade of EMAP carried out in 2007 permits exposure dose calculations.

\section{Main features of BERS}

BERS consists of two parts - operational and accidental ones.

The operational part runs automatically each day a fixed moment after receiving the new meteorological information via the global telecomunication sustem of the World Meteorological Organization. For the purpose the product of the Global model of the German Weather Service is used (resolution $1.5^{\circ}$ in space and $12 \mathrm{~h}$ in time). The operational chain includes several modules performing: (i) preparation of specific internal meteorological file used subsequentially by both a trajectory model and the dispersion model EMAP; (ii) archiving this meteorological data for further resimulations; (iii) running of trajectory model; (iv) preparing the visualization of results and uploading the trajectory maps to a specialized web site of $\mathrm{NIMH}$. 
The 3-day forward trajectories are calculated for 35 European NPPs. For each NPP a set of 3 trajectories is prepared with release heights of 100,300 and $1000 \mathrm{~m}$. This data is for information of Bulgarian emergency authorities about the possible transfer over the country in case of nuclear accident.

The accidental part is activated by an operator after request from the emergency authorities that shall provide the necessary source information - coordinates and release parameters (start and end time, height, duration, rate etc.). This information, together with the internal meteorological file, is input to EMAP model. EMAP is capable to operate with changing parameters of the release as well. The model outputs in this case are time sequences of concentration and deposition fields. They are visualised (eventually animated) and provided to the user.

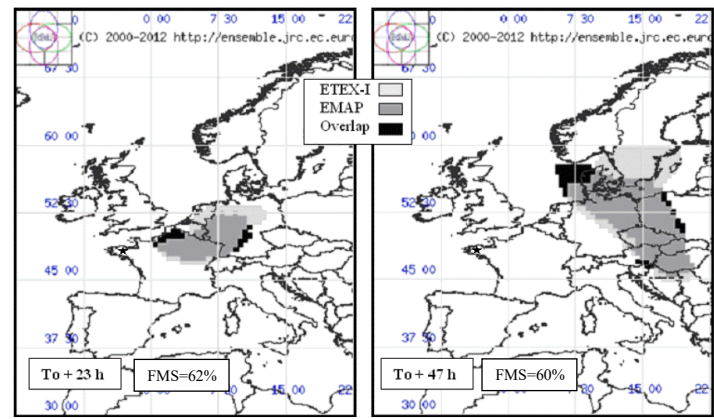

Fig. 1. Overlapping of measured and simulated by EMAP tracer spots for two moments after the release start to (a star indicates the release position).
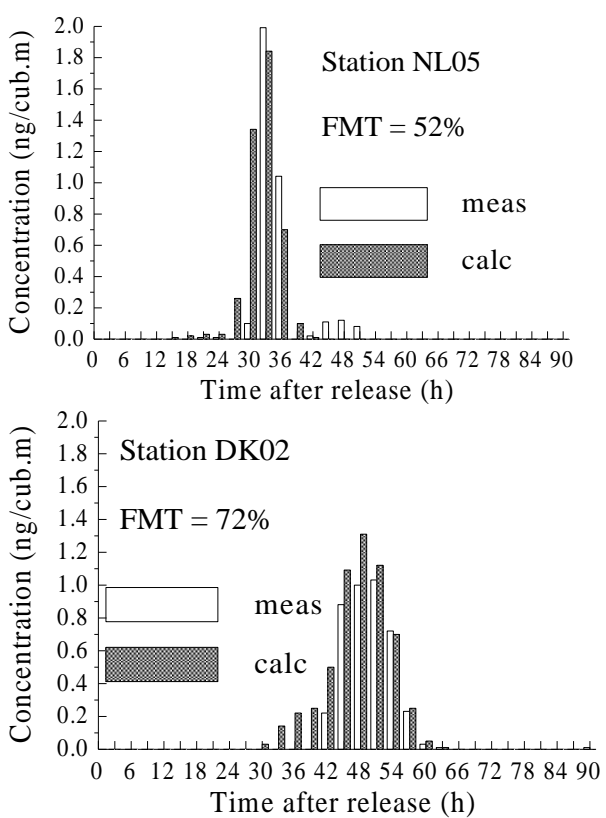

Fig. 2. Time evolution of measured and calculated concentrations in two measuring stations in Netherlands (NLO5) and in Denmark (DK02).
All this calculations (trajectory and dispersion) are performed over area covering Europe using $250 \times 210$ grid points with $20 \mathrm{~km}$ resolution (Lambert projection).

\section{EMAP model}

EMAP (Eulerian Model for Air Pollution) is the core of BERS.

It is 3-dimentional with irregular grid in the vertical. The processes of advection (transportation), diffusion, dry \& wet deposition, and chemical or radioactive transformations are accounted for by means of not very complicated numerical schemes. Nevertheless, the validation in the frame of ETEX project shows that the model simulates the real release pretty well as can be seen from Fig. 1 and Fig. 2 .

In the figures, two important parameters are estimated - Figure of Merit in Space (FMS) and Figure of Merit in Time (FMT). Both parameters point out a good quality of the simulation. The complicated ranging performed in Graziani et al. (1998), shows that EMAP results are rated 9th among 34 models participating in ETEX.

\section{The new version of BERS (BERS2)}

The development of a new, updated version of BERS was driven by many factors - the increase of computational abilities of the new generation of computers, the appearance of new open access data bases, the availability of new free of charge comprehensive meteorological models, the improved high speed of downloading and other internet operations etc. The main modifications concern the operational part of the system.

The idea is first to create new improved internal meteorological file using data with higher resolution in space and time. The present day world meteorological centers produce meteorological forecast with high reliability, i.e. the skill score of the important for dispersion calculations meteorological parameters like wind, temperature, humidity, precipitation is quite good. It is especially important to have good precipitation distribution and intensity as this is considered crucial for the cleaning of the atmosphere by removing the pollution from the air.

The USA National Centers for Environmental Prediction (NCEP) meteorological forecast provided by its Global Forecast System (GFS) was chosen for the purpose. Recent improvements in the GFS web-site allow the user to access data with much higher resolution $\left(0.25^{\circ}\right.$ in space and $6 \mathrm{~h}$ in time) for any specified area over the globe. Together with the improved forecast skill of the meteorological 
parameters, the new space resolution is of the order of the grid step of our computational mesh, i.e. the interpolation in the grid point will lead to smaller errors and will reflect better the small-scale peculiarities in met-parameter's distribution.

The second idea is to enhance the quantity of information that would be useful to the emergency authorities. The previous version of BERS provides only forecasted trajectories, which reflect only one part of the dispersion processes, mainly the advection. In the new version, the possible distribution of pollutants in space and time is also provided. For that purpose it was decided to perform EMAP calculations with the new improved meteorological data and fictitious source parameters simulating a serious nuclear accident.
The modelled concentration and deposition fields could be visualized, animated and presented in a respective web-page together with the trajectory images.

Linux environment is required as to be able to access and to maintain the new meteorological data needed for BERS2. The GFS met data, presented in GRIB format, are not directly interpolated in the grid points of BERS2 mesh. Instead, dynamical interpolation is performed by means of the sophisticated mesometeorological model WRF (Weather Research Forecast). Its output has 1-hour time resolution and is further processed with the MCIP (Meteorology-Chemistry interface Processor); both model, important part of the US EPA (https://www.cmascenter.org) Models-3 system. Both models work in multi-processor regime.

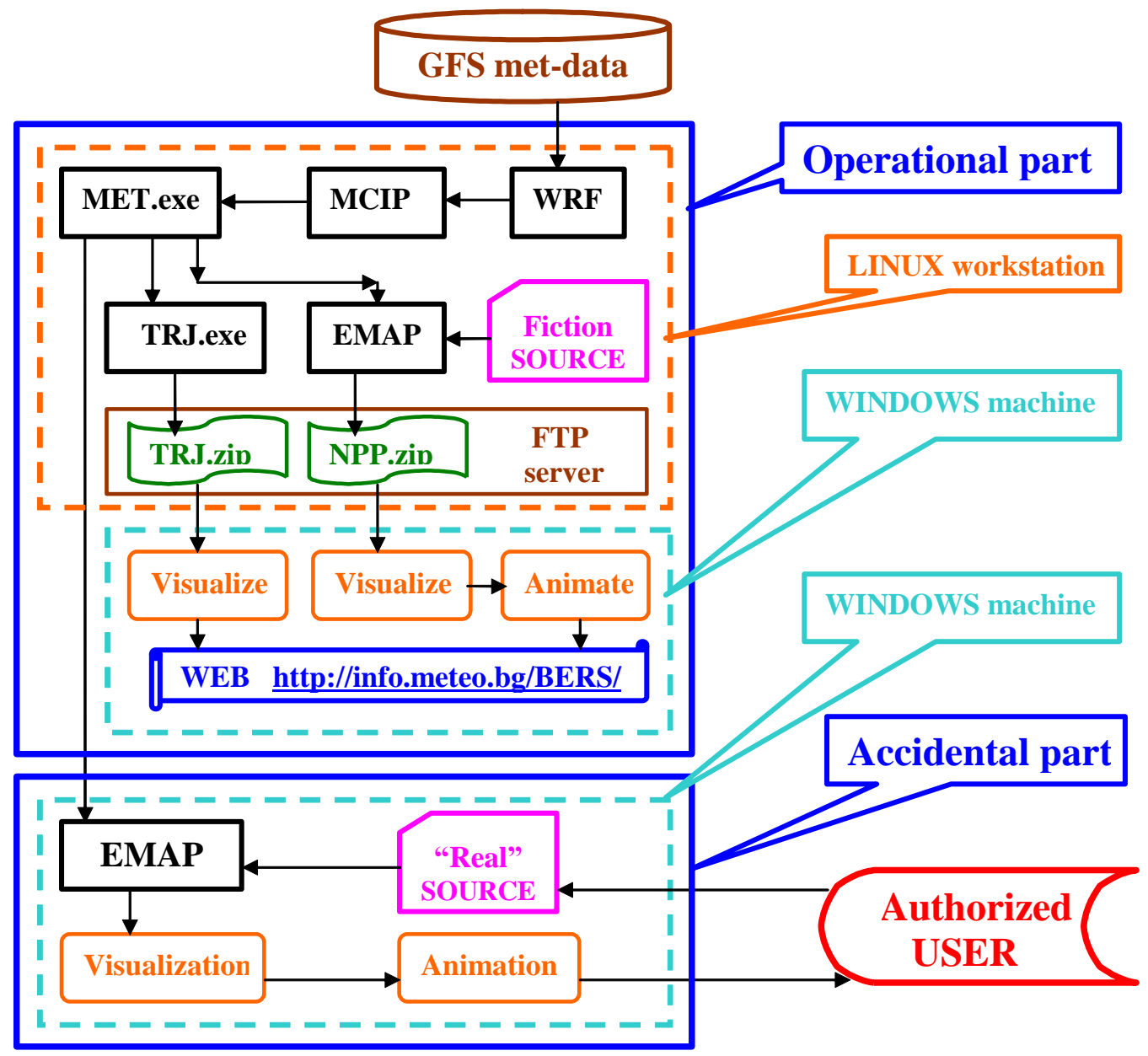

Fig.3. Data flow chart of BERS2.

In Fig.3, the BERS2 flow chart is presented, showing the sequence of operations in both parts. The MCIP output feeds the MET.exe module that produces the internal met-file. It is input to the trajectory model TRJ.exe and to EMAP (in both the operational and the accidental parts of the System). The trajectory and dispersion outputs are packed and uploaded to a dedicated FTP server.

The visualization is performed by a Windows machine. It is activated automatically and downloads the packed files, first. Then, trajectory files are processed producing 6 
images with trajectories from 6 different NPPs, all images uploaded to the BERS2 web-page. The concentration and deposition fields are further visualized and animated. The respective images are also uploaded to the same webpage.
The accidental part was not significantly changed. First, the operator prepares the source input to EMAP. The met-input is already prepared and saved in the Windows machine. The EMAP output is treated respectively.

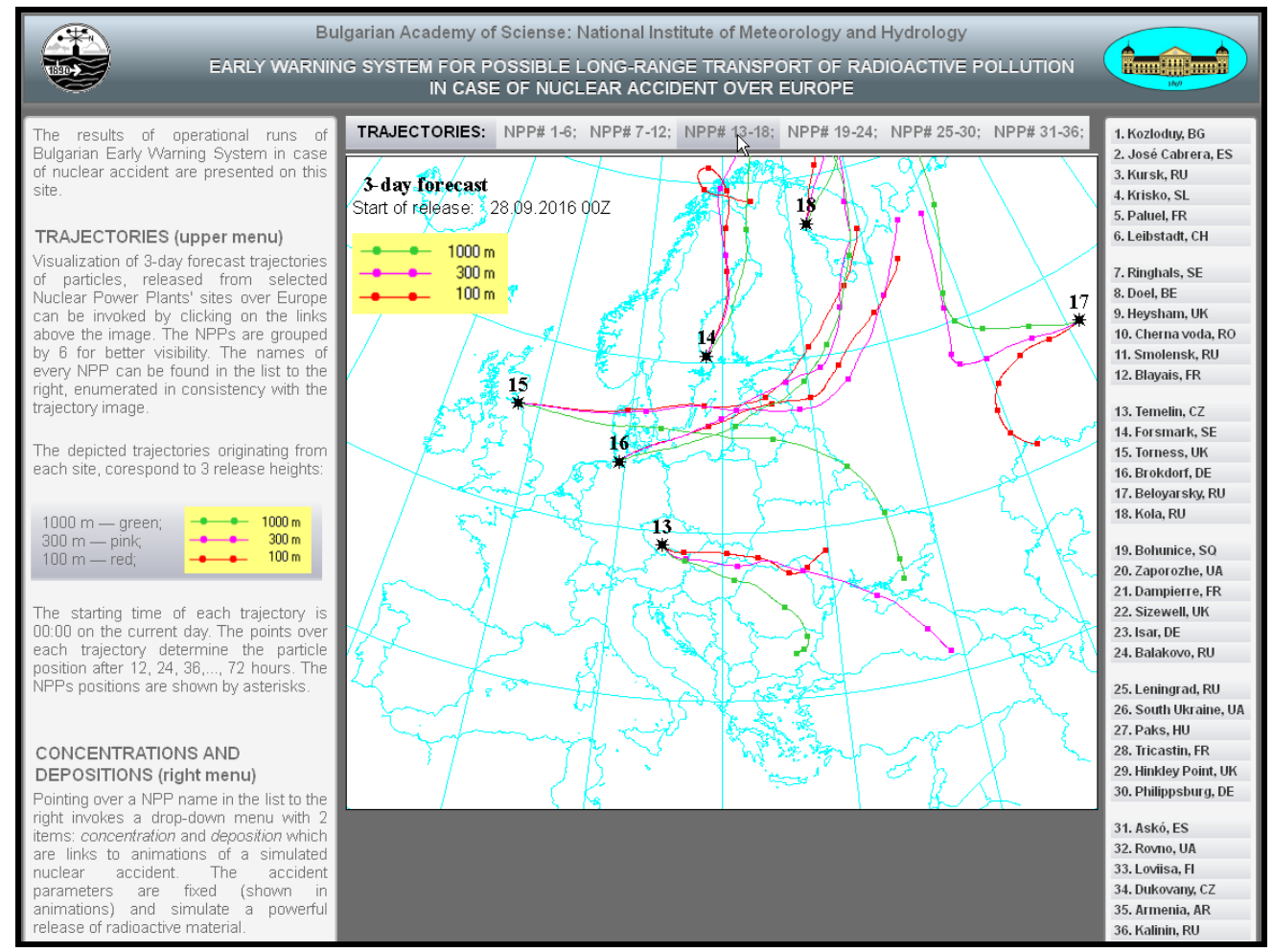

Fig. 4. View of BERS2 web-page (trajectory case).

\section{BERS2 web-page http://info.meteo.bg/BERS}

The increased quantity of information required the creating of a new web page (Fig. 4.). It consists of four parts. In the left column, short description of the data is shown together with hints how to invoke the various type of information. A menu list with trajectory images is posed on the top where the groups of NPPs are denoted. A mouse click over an item invokes appearance of respective image in the central part of the page.

The right column contains a menu list of 36 European Nuclear Power Plants, divided into 6 groups. This column plays twofold role. First, it explains the trajectory images, where each NPP is denoted by a number only. Secondly, it allows invoking animations for particular NPP. Posing the pointer over the required NPP, a drop-down menu appears with "concentration" and "deposition". Click over each of them activates the respective animation in the central part of the page. The factious source parameters are shown right of this image.

\section{Conclusion}

The increasing variety and quantity of information for the possible transport of radioactive pollution in case of nuclear accident provided by the new version of BERS can be very helpful for emergency authorities.

\section{References}

[1] Graziani, G., W. Klug, S. Moksa. Editors. (1998): Real-Time Long-Range Dispersion Model Evaluation of the ETEX First Release. Joint Research Centre, EU, Luxemburg.

[2] Syrakov, D. and Prodanova, M. (1998): Bulgarian Emergency Response Models - Validation against ETEX First Release, Atmospheric Environment, 32, 4367-4375.

[3] Syrakov D., Prodanova M. and K. Slavov (2003): Description and performance of Bulgarian Emergency Response System in case of nuclear accident (BERS), Int. J. Environment and Pollution, 20, 1-6. 